\section{ISEB and EMBnet to collaborate on bioinformatics education and training}

\section{ISCB Press Release}

SAN DIEGO, USA and UPPSALA, SWEDEN - 26 April 2011 - The International Society for Computational Biology (ISCB) and the European Molecular Biology Network (EMBnet) are pleased to announce a collaboration to provide education and training in the use of bioinformatics tools to members of our communities. Having just sponsored and organised a successful workshop introducing the EMBnet eBioKit at the ISCB Africa ASBCB Conference on Bioinformatics 2011 (www.iscb.org/iscbafrica2011/), that followed a similarly sponsored and organised workshop on sequence analysis using EMBOSS at the 2009 rendition of the same conference (www.iscb. org/iscbafrica2009/), both EMBnet Executive Board member Erik Bongcam-Rudloff and ISCB President Burkhard Rost enthusiastically embraced the idea of a formal collaboration that will ensure EMBnet training courses are incorporated into ISCB meetings whenever possible, particularly those in developing regions.

"EMBnet is a very strong member of ISCB's Affiliated Regional Network, and formalizing our collaboration to incorporate their training into ISCB conferences makes perfect sense," commented Prof. Dr. Rost. "They do this very well and our member community will undoubtedly benefit from their expertise," he continued. The next meeting that may be targeted for inclusion of an EMBnet training workshop is the recently announced InCoB/ISCB-Asia conference taking place in Kuala Lumpur, Malaysia, November 27December 2, 2011. Following that, discussion has already begun on incorporating an EMBnet training session in the next ISCB-Latin America conference, to be held in Santiago, Chile, in March 2012.

As EMBnet is comprised of 28 national and eight specialists nodes, strong ties to many of the active bioinformaticians in countries around the world are securely in place. Prof. Dr. BongcamRudloff summed up the collaboration nicely: "This is a natural fit. ISCB is expanding its regional meetings into areas where EMBnet has close working relationships with high-level members of our network. Working together we will accomplish more, and everybody wins."

EMBnet has also worked closely with ISCB's Student Council, featuring their accomplishments in the EMBnet Journal, providing technical help with a virtual meeting organised by the students, and presenting an eBioKit tutorial to a joint meeting of several Regional Student Groups. Both Bongcam-Rudloff and Rost agree: "This collaboration establishes the framework for further involvement of EMBnet in ISCB Student Council activities. This is good for the future of our entire scientific community."

The International Society for Computational Biology (http://www.iscb.org) is the leading professional society for the new era of computational biology.

For further information contact info@iscb.org

EMBnet (http://www.embnet.org) is a sciencebased group of collaborating nodes throughout all continents.

For further information contact Erik.Bongcam@ slu.se 